\title{
Supplementary materials: Gaussian orthogonal latent factor processes for large incomplete matrices of correlated data
}

\author{
Mengyang $\mathrm{Gu}^{*}$ and Hanmo $\mathrm{Li}^{\dagger}$
}

This supplementary materials contain three parts. The proof of Section 2 is given in Section S1. The additional numerical results for the simulated studies and real applications are given in Section S2 and Section S3, respectively.

\section{S1 Proofs for Section 2}

\section{S1.1 Auxiliary facts}

1. Let $\mathbf{A}$ and $\mathbf{B}$ be matrices,

$$
(\mathbf{A} \otimes \mathbf{B})^{T}=\left(\mathbf{A}^{T} \otimes \mathbf{B}^{T}\right) ;
$$

further assuming $\mathbf{A}$ and $\mathbf{B}$ are invertible,

$$
(\mathbf{A} \otimes \mathbf{B})^{-1}=\mathbf{A}^{-1} \otimes \mathbf{B}^{-1} .
$$

2. Let $\mathbf{A}, \mathbf{B}, \mathbf{C}$ and $\mathbf{D}$ be the matrices such that the products $\mathbf{A C}$ and $\mathbf{B D}$ are matrices,

$$
(\mathbf{A} \otimes \mathbf{B})(\mathbf{C} \otimes \mathbf{D})=(\mathbf{A C}) \otimes(\mathbf{B D}) .
$$

3. For matrices $\mathbf{A}, \mathbf{B}$ and $\mathbf{C}$,

$$
\left(\mathbf{C}^{T} \otimes \mathbf{A}\right) \operatorname{vec}(\mathbf{B})=\operatorname{vec}(\mathbf{A B C}) ;
$$

further assuming $\mathbf{A}^{T} \mathbf{B}$ is a matrix,

$$
\operatorname{tr}\left(\mathbf{A}^{T} \mathbf{B}\right)=\operatorname{vec}(\mathbf{A})^{T} \operatorname{vec}(\mathbf{B}) .
$$

4. For any invertible $n \times n$ matrix $\mathbf{C}$,

$$
|\mathbf{C}+\mathbf{A B}|=|\mathbf{C}|\left|\mathbf{I}_{n}+\mathbf{B C}^{-1} \mathbf{A}\right| .
$$

*Department of Statistics and Applied Probability, University of California, Santa Barbara, CA mengyang@pstat.ucsb.edu

${ }^{\dagger}$ Department of Statistics and Applied Probability, University of California, Santa Barbara, CA hanmo@pstat.ucsb.edu 


\section{S1.2 Proofs for Section 2.1}

The following denotation are used in the proof: $\mathbf{Y}_{-M}=\mathbf{Y}-\mathbf{M}, \mathbf{Y}_{v,-M}=\operatorname{vec}(\mathbf{Y}-\mathbf{M})$, $\mathbf{Z}_{v t}=\operatorname{vec}\left(\mathbf{Z}^{T}\right)$ and $\mathbf{A}_{v}=\left[\mathbf{I}_{n_{2}} \otimes \mathbf{a}_{1}, \ldots, \mathbf{I}_{n_{2}} \otimes \mathbf{a}_{d}\right]$. Let $\boldsymbol{\Sigma}_{v}$ be an $n_{2} d \times n_{2} d$ matrix where the $l$ th diagonal block is $\boldsymbol{\Sigma}_{l}$. Denote $\operatorname{etr}()=.\exp (\operatorname{tr}()$.$) .$

Proof of Equation 2.4. Denote $C_{Y}=\left(2 \pi \sigma_{0}^{2}\right)^{-\frac{n_{1} n_{2}}{2}} \prod_{l=1}^{d}\left|\boldsymbol{\Sigma}_{l} / \sigma_{0}^{2}+\mathbf{I}_{n_{2}}\right|^{-1 / 2}$. Directly marginalizing out $\mathbf{Z}$, one has

$$
\begin{aligned}
& p(\mathbf{Y} \mid \boldsymbol{\Theta}) \\
= & C_{Y} \exp \left(-\frac{\mathbf{Y}_{v,-M}^{T}\left(\mathbf{I}_{n_{1} n_{2}}-\sum_{l=1}^{d}\left(\sigma_{0}^{2} \boldsymbol{\Sigma}_{l}^{-1}+\mathbf{I}_{n_{2}}\right)^{-1} \otimes\left(\mathbf{a}_{l} \mathbf{a}_{l}^{T}\right)\right) \mathbf{Y}_{v,-M}}{2 \sigma_{0}^{2}}\right) \\
= & C_{Y} \exp \left(-\frac{\mathbf{Y}_{v,-M}^{T} \mathbf{Y}_{v,-M}-\mathbf{Y}_{v,-M}^{T} \sum_{l=1}^{d} \operatorname{vec}\left(\mathbf{a}_{l} \mathbf{a}_{l}^{T} \mathbf{Y}_{v,-M}\left(\sigma_{0}^{2} \boldsymbol{\Sigma}_{l}^{-1}+\mathbf{I}_{n_{2}}\right)^{-1}\right)}{2 \sigma_{0}^{2}}\right) \\
= & C_{Y} \operatorname{etr}\left(-\frac{\mathbf{Y}_{-M}^{T} \mathbf{Y}_{-M}-\sum_{l=1}^{d} \tilde{\mathbf{y}}_{l} \tilde{\mathbf{y}}_{l}^{T}\left(\sigma_{0}^{2} \boldsymbol{\Sigma}_{l}^{-1}+\mathbf{I}_{n_{2}}\right)^{-1}}{2 \sigma_{0}^{2}}\right) \\
= & C_{Y} \exp \left(-\frac{\sum_{l=1}^{d} \tilde{\mathbf{y}}_{l}^{T}\left(\boldsymbol{\Sigma}_{l} / \sigma_{0}^{2}+\mathbf{I}_{n_{2}}\right)^{-1} \tilde{\mathbf{y}}_{l}+\sum_{l=d+1}^{n_{1}} \tilde{\mathbf{y}}_{l}^{T} \tilde{\mathbf{y}}_{l}}{2 \sigma_{0}^{2}}\right),
\end{aligned}
$$

where the first equation is based on Lemma 1 and the Woodbury matrix identity (to compute the normalizing constant $C_{Y}$ ); the second and third equations are from fact 3 ; the fourth equation is from Woodbury matrix identity. The Equation (2.4) follows immediately.

Proof of Corollary 1. The proof is implied by the proof of Theorem 4 in (Gu and Shen, 2020). For completeness of this article, we include the proof below.

From Equation (2.1) and Equation (2.2), we have

$$
\begin{aligned}
p\left(\mathbf{Z}_{v t} \mid \mathbf{Y}, \boldsymbol{\Theta}\right) & \propto \exp \left(\frac{\left(\mathbf{Y}_{v,-M}-\mathbf{A}_{v} \mathbf{Z}_{v t}\right)^{T}\left(\mathbf{Y}_{v,-M}-\mathbf{A}_{v} \mathbf{Z}_{v t}\right)}{2 \sigma_{0}^{2}}\right) \exp \left(-\frac{1}{2} \mathbf{Z}_{v t}^{T} \boldsymbol{\Sigma}_{v}^{-1} \mathbf{Z}_{v t}\right) \\
& \propto \exp \left\{-\frac{1}{2}\left(\mathbf{Z}_{v t}-\boldsymbol{\mu}_{Z_{v t}}\right)^{T}\left(\frac{\mathbf{A}_{v}^{T} \mathbf{A}_{v}}{\sigma_{0}^{2}}+\boldsymbol{\Sigma}_{v}^{-1}\right)\left(\mathbf{Z}_{v t}-\boldsymbol{\mu}_{Z_{v t}}\right)\right\},
\end{aligned}
$$

where $\boldsymbol{\mu}_{Z_{v t}}=\left(\mathbf{A}_{v}^{T} \mathbf{A}_{v}+\sigma_{0}^{2} \boldsymbol{\Sigma}_{v}^{-1}\right)^{-1} \mathbf{A}_{v}^{T} \mathbf{Y}_{v,-M}$. Note $\mathbf{A}_{v}^{T} \mathbf{A}_{v}=\mathbf{I}_{n_{2} d}$, from which we have

$$
\mathbf{Z}_{v t} \mid \mathbf{Y}, \boldsymbol{\Theta} \sim \mathrm{MN}\left(\boldsymbol{\mu}_{Z_{v t}},\left(\frac{1}{\sigma_{0}^{2}} \mathbf{I}_{n_{2} d}+\boldsymbol{\Sigma}_{v}^{-1}\right)^{-1}\right)
$$


Based on vectorization, one has

$$
\begin{aligned}
\boldsymbol{\mu}_{Z_{v t}}=\left(\begin{array}{c}
\left(\sigma_{0}^{2} \boldsymbol{\Sigma}_{1}^{-1}+\mathbf{I}_{n_{2}}\right)^{-1} \otimes \mathbf{a}_{1}^{T} \\
\vdots \\
\left(\sigma_{0}^{2} \boldsymbol{\Sigma}_{d}^{-1}+\mathbf{I}_{n_{2}}\right)^{-1} \otimes \mathbf{a}_{d}^{T}
\end{array}\right)^{\operatorname{vec}(\mathbf{Y})=\left(\begin{array}{c}
\operatorname{vec}\left(\mathbf{a}_{1}^{T} \mathbf{Y}_{-M}\left(\sigma_{0}^{2} \boldsymbol{\Sigma}_{1}^{-1}+\mathbf{I}_{n_{2}}\right)^{-1}\right) \\
\vdots \\
\operatorname{vec}\left(\mathbf{a}_{d}^{T} \mathbf{Y}_{-M}\left(\sigma_{0}^{2} \boldsymbol{\Sigma}_{d}^{-1}+\mathbf{I}_{n_{2}}\right)^{-1}\right)
\end{array}\right)} \\
=\operatorname{vec}\left(\begin{array}{c}
\mathbf{a}_{1}^{T} \mathbf{Y}_{-M}\left(\sigma_{0}^{2} \boldsymbol{\Sigma}_{1}^{-1}+\mathbf{I}_{n_{2}}\right)^{-1} \\
\vdots \\
\mathbf{a}_{d}^{T} \mathbf{Y}_{-M}\left(\sigma_{0}^{2} \boldsymbol{\Sigma}_{d}^{-1}+\mathbf{I}_{n_{2}}\right)^{-1}
\end{array}\right)^{T} .
\end{aligned}
$$

Note that the covariance matrix of $\boldsymbol{\mu}_{Z_{v t}}$ is a block diagonal matrix. The results follow by Equation (S2) and the Woodbury matrix identity.

\section{S1.3 Proofs for Section 2.2}

Note $\mathbf{A}_{F}=\left[\mathbf{A}_{s}, \mathbf{A}_{c}\right]=\left[\mathbf{a}_{1}, \mathbf{a}_{2}, \ldots, \mathbf{a}_{n_{1}}\right]$, where $\mathbf{A}_{c}$ is an $n_{1} \times\left(n_{1}-d\right)$ matrix of the orthogonal complement of $\mathbf{A}_{s}$. We need the following lemma to prove Theorem 1 .

Lemma S1. After marginalizing out the factors $\mathbf{Z}$, we have the marginal posterior distribution of the transformed regression coefficients,

1. (Marginal distribution of transformed row regression coefficients). Assume $\mathbf{M}=$ $\mathbf{H}_{1} \mathbf{B}_{1}$ and the objective prior $\pi\left(\mathbf{B}_{1}\right) \propto 1$ for $\mathbf{B}_{1}$. Let $\tilde{\mathbf{B}}_{1}=\left[\tilde{\boldsymbol{b}}_{1,1}, \ldots, \tilde{\boldsymbol{b}}_{1, n_{1}}\right]=$ $\mathbf{B}_{1}^{T} \mathbf{H}_{1}^{T} \mathbf{A}_{F}$ be an $n_{2} \times n_{1}$ matrix of transformed coefficients. Assume the marginal posterior distribution of $\tilde{\mathbf{B}}_{1}$ follows

$$
p\left(\tilde{\mathbf{B}}_{1} \mid \mathbf{Y}, \boldsymbol{\Theta}_{-B_{1}}\right)=\prod_{l=1}^{d} \mathcal{P} \mathcal{N}\left(\tilde{\boldsymbol{b}}_{1, l} ; \tilde{\mathbf{y}}_{l}, \tilde{\boldsymbol{\Sigma}}_{l}\right) \prod_{l=d+1}^{n_{1}} \mathcal{P} \mathcal{N}\left(\tilde{\boldsymbol{b}}_{1, l} ; \tilde{\mathbf{y}}_{l}, \sigma_{0}^{2} \mathbf{I}_{n_{2}}\right)
$$

where $\tilde{\mathbf{y}}_{l}$ is defined in equation (2.4) and $\tilde{\boldsymbol{\Sigma}}_{l}$ is defined in corollary 1. Then we can sample $\left(\mathbf{B}_{1} \mid \mathbf{Y}, \mathbf{\Theta}_{-B_{1}}\right)$ by $\left(\mathbf{H}_{1}^{T} \mathbf{H}_{1}\right)^{-1} \mathbf{H}_{1}^{T} \mathbf{A}_{F} \tilde{\mathbf{B}}_{\mathbf{1}}^{T}$, where $\tilde{\mathbf{B}}_{\mathbf{1}}^{T}$ are sampled from the $p\left(\tilde{\mathbf{B}}_{1} \mid \mathbf{Y}, \boldsymbol{\Theta}_{-B_{1}}\right)$ in equation (S3).

2. (Marginal distribution of transformed column regression coefficients). Assume $\mathbf{M}=$ $\left(\mathbf{H}_{2} \mathbf{B}_{2}\right)^{T}$ and the objective prior $\pi\left(\mathbf{B}_{2}\right) \propto 1$ for the regression parameters $\mathbf{B}_{2}$. Let $\tilde{\mathbf{B}}_{2}=\left[\tilde{\boldsymbol{b}}_{2,1}, \ldots, \tilde{\boldsymbol{b}}_{2, n_{1}}\right]=\mathbf{B}_{2} \mathbf{A}_{F}$ be a $q_{2} \times n_{1}$ matrix. The marginal posterior distribution of $\tilde{\mathbf{B}}_{2}$ follows

$$
p\left(\tilde{\mathbf{B}}_{2} \mid \mathbf{Y}, \boldsymbol{\Theta}_{-B_{2}}\right)=\prod_{l=1}^{n_{1}} \mathcal{P} \mathcal{N}\left(\tilde{\mathbf{b}}_{2, l} ; \boldsymbol{\mu}_{\tilde{b}_{2, l}}, \boldsymbol{\Sigma}_{\tilde{b}_{2, l}}\right)
$$

where $\boldsymbol{\mu}_{\tilde{b}_{2, l}}=\left(\mathbf{H}_{2}^{T} \tilde{\boldsymbol{\Sigma}}_{l}^{-1} \mathbf{H}_{2}\right)^{-1} \mathbf{H}_{2}^{T} \tilde{\boldsymbol{\Sigma}}_{l}^{-1} \tilde{\mathbf{y}}_{l}$ and $\boldsymbol{\Sigma}_{\tilde{b}_{2, l}}=\left(\mathbf{H}_{2}^{T} \tilde{\boldsymbol{\Sigma}}_{l}^{-1} \mathbf{H}_{2}\right)^{-1}$ for $l=$ $1, \ldots, d ; \boldsymbol{\mu}_{\tilde{b}_{2, l}}=\left(\mathbf{H}_{2}^{T} \mathbf{H}_{2}\right)^{-1} \mathbf{H}_{2}^{T} \tilde{\mathbf{y}}_{l}$ and $\boldsymbol{\Sigma}_{\tilde{b}_{2, l}}=\sigma_{0}^{2}\left(\mathbf{H}_{2}^{T} \mathbf{H}_{2}\right)^{-1}$ for $l=d+1, \ldots, n_{1}$. 
Proof of Lemma S1. 1. (Marginal distribution of transformed row regression coefficients).

Denote $\left(\mathbf{B}_{1}^{\text {aug }}\right)=\left[\mathbf{B}_{1}^{T}, \tilde{\mathbf{B}}_{1,\left(q_{1}+1\right): n_{1}}\right]^{T}$, where $\tilde{\mathbf{B}}_{1,\left(q_{1}+1\right): n_{1}}$ are the last $n_{1}-q_{1}$ columns of $\tilde{\mathbf{B}}_{1}$. Denote $p_{\text {trans }}\left(\mathbf{B}_{1} \mid \mathbf{Y}, \boldsymbol{\Theta}_{-\mathbf{B}_{1}}\right)$ and $p_{\text {trans }}\left(\mathbf{B}_{1}^{\text {aug }} \mid \mathbf{Y}, \boldsymbol{\Theta}_{-\mathbf{B}_{1}}\right)$ the transformed marginal posterior distribution of $\mathbf{B}_{1}$ and $\mathbf{B}_{1}^{\text {aug }}$ derived by transforming $p\left(\tilde{\mathbf{B}}_{1} \mid \mathbf{Y}, \boldsymbol{\Theta}_{-B_{1}}\right)$ in (S3). We have

$$
\begin{gathered}
p_{\text {trans }}\left(\mathbf{B}_{1} \mid \mathbf{Y}, \boldsymbol{\Theta}_{-\mathbf{B}_{1}}\right) \propto p_{\text {trans }}\left(\mathbf{B}_{1}^{\text {aug }} \mid \mathbf{Y}, \boldsymbol{\Theta}_{-\mathbf{B}_{1}}\right)=p\left(\tilde{\mathbf{B}}_{1} \mid \mathbf{Y}, \boldsymbol{\Theta}_{-\mathbf{B}_{1}}\right)\left|\frac{d \tilde{\mathbf{B}}_{1}}{d \mathbf{B}_{1}^{\text {aug }}}\right| \\
\propto \exp \left\{-\frac{1}{2} \sum_{l=1}^{d}\left(\tilde{\mathbf{b}}_{1, l}-\tilde{\mathbf{y}}_{l}\right)^{T} \tilde{\boldsymbol{\Sigma}}_{l}^{-1}\left(\tilde{\mathbf{b}}_{1, l}-\tilde{\mathbf{y}}_{l}\right)-\frac{1}{2 \sigma_{0}^{2}} \sum_{l=d+1}^{n_{1}}\left(\tilde{\boldsymbol{b}}_{1, l}-\tilde{\mathbf{y}}_{l}\right)^{T}\left(\tilde{\boldsymbol{b}}_{1, l}-\tilde{\mathbf{y}}_{l}\right)\right\} \\
\propto \exp \left\{-\frac{1}{2} \sum_{l=1}^{d} \mathbf{a}_{l}^{T}\left(\mathbf{Y}-\mathbf{H}_{1} \mathbf{B}_{1}\right) \tilde{\boldsymbol{\Sigma}}_{l}^{-1}\left(\mathbf{Y}-\mathbf{H}_{1} \mathbf{B}_{1}\right)^{T} \mathbf{a}_{l}\right. \\
\left.-\frac{1}{2 \sigma_{0}^{2}} \sum_{l=d+1}^{n_{1}} \mathbf{a}_{l}^{T}\left(\mathbf{Y}-\mathbf{H}_{1} \mathbf{B}_{1}\right)\left(\mathbf{Y}-\mathbf{H}_{1} \mathbf{B}_{1}\right)^{T} \mathbf{a}_{l}\right\},
\end{gathered}
$$

where the last line is the same as the posterior distribution of $\mathbf{B}_{1}$ based on the marginal likelihood in equation (2.4) and the prior distribution $\pi\left(\mathbf{B}_{1}\right) \propto 1$. Thus if one sample $\tilde{\mathbf{B}}_{1}$ from (S3), one can obtain the sample for $\left(\mathbf{B}_{1} \mid \mathbf{Y}, \boldsymbol{\Theta}_{-\mathbf{B}_{1}}\right)$ through $\left(\mathbf{H}_{1}^{T} \mathbf{H}_{1}\right)^{-1} \mathbf{H}_{1}^{T} \mathbf{A}_{F} \tilde{\mathbf{B}}_{\mathbf{1}}^{T}$.

2. (Marginal distribution of transformed column regression coefficients).

Since $\pi\left(\mathbf{B}_{2}\right) \propto 1$ is a Jeffreys prior, and $\tilde{\mathbf{B}}_{2}$ is a linear transformation of $\mathbf{B}_{2}$ with the same dimension, we have $\pi\left(\tilde{\mathbf{B}}_{2}\right) \propto 1$.

Based on the marginal likelihood in equation (2.4) and the prior distribution, the posterior distribution of $\tilde{\mathbf{B}}_{2}$ follows:

$$
\begin{aligned}
& \quad p\left(\tilde{\mathbf{B}}_{2} \mid \mathbf{Y}, \boldsymbol{\Theta}_{-\mathbf{B}_{2}}\right) \\
& \propto \exp \left\{-\frac{1}{2} \sum_{l=1}^{d} \mathbf{a}_{l}^{T}\left(\mathbf{Y}-\mathbf{B}_{2}^{T} \mathbf{H}_{2}^{T}\right) \tilde{\boldsymbol{\Sigma}}_{l}^{-1}\left(\mathbf{Y}-\mathbf{B}_{2}^{T} \mathbf{H}_{2}^{T}\right)^{T} \mathbf{a}_{l}\right. \\
& \left.\quad-\frac{1}{2 \sigma_{0}^{2}} \sum_{l=d+1}^{n_{1}} \mathbf{a}_{l}^{T}\left(\mathbf{Y}-\mathbf{B}_{2}^{T} \mathbf{H}_{2}^{T}\right)\left(\mathbf{Y}-\mathbf{B}_{2}^{T} \mathbf{H}_{2}^{T}\right)^{T} \mathbf{a}_{l}\right\} \\
& \propto \exp \left\{-\frac{1}{2} \sum_{l=1}^{d}\left(\tilde{\mathbf{y}}_{l}-\mathbf{H}_{2} \tilde{\mathbf{b}}_{2, l}\right)^{T} \tilde{\boldsymbol{\Sigma}}_{l}^{-1}\left(\tilde{\mathbf{y}}_{l}-\mathbf{H}_{2} \tilde{\mathbf{b}}_{2, l}\right)\right. \\
& \propto \exp \left\{-\frac{1}{2 \sigma_{0}^{2}} \sum_{l=d+1}^{n_{1}}\left(\tilde{\mathbf{y}}_{l}-\mathbf{H}_{2} \tilde{\mathbf{b}}_{2, l}\right)^{T}\left(\tilde{\mathbf{y}}_{l}-\mathbf{H}_{2} \tilde{\mathbf{b}}_{2, l}\right)\right\} \\
&
\end{aligned}
$$




$$
\left.-\frac{1}{2 \sigma_{0}^{2}} \sum_{l=d+1}^{n_{1}}\left(\tilde{\mathbf{b}}_{2, l}-\boldsymbol{\mu}_{\tilde{b}_{2, l}}\right)^{T} \mathbf{H}_{2}^{T} \mathbf{H}_{2}\left(\tilde{\mathbf{b}}_{2, l}-\boldsymbol{\mu}_{\tilde{b}_{2, l}}\right)\right\},
$$

from which equation (S4) follows.

We are ready to prove Theorem 1 .

Proof of Theorem 1. After marginalizing out $\mathbf{Z}$, we have

1. (Row regression coefficients).

From Lemma S1, the posterior mean of $\left(\tilde{\mathbf{B}}_{1} \mid \mathbf{Y}, \boldsymbol{\Theta}_{-\mathbf{B}_{1}}\right)$ is $\mathbf{Y}^{T} \mathbf{A}_{F}$, where $\mathbf{A}_{F}:=$ $\left[\mathbf{A}_{s}, \mathbf{A}_{c}\right]$. We denote the centered $\tilde{\mathbf{B}}_{1}$ by $\tilde{\mathbf{B}}_{1,0}=\left[\tilde{\mathbf{B}}_{1,0, s}, \tilde{\mathbf{B}}_{1,0, c}\right]=\tilde{\mathbf{B}}_{1}-\mathbf{Y}^{T} \mathbf{A}_{F}$, where $\tilde{\mathbf{B}}_{1,0, s}$ is the first $d$ columns of $\tilde{\mathbf{B}}_{1,0}$ and $\tilde{\mathbf{B}}_{1,0, c}$ is the last $\left(n_{1}-d\right)$ columns of $\tilde{\mathbf{B}}_{1,0}$. Let $\tilde{\mathbf{b}}_{1,0, l}$ be the $l$-th column of $\tilde{\mathbf{B}}_{1,0}$. Then the posterior mean of $\left(\mathbf{B}_{1} \mid\right.$ $\mathbf{Y}, \boldsymbol{\Theta}_{-\mathbf{B}_{1}}$ ) can be calculated below

$$
\begin{aligned}
\hat{\mathbf{B}}_{1} & =\mathbb{E}\left(\mathbf{B}_{1} \mid \mathbf{Y}, \boldsymbol{\Theta}_{-\mathbf{B}_{1}}\right)=\mathbb{E}\left(\left(\mathbf{H}_{1}^{T} \mathbf{H}_{1}\right)^{-1} \mathbf{H}_{1}^{T} \mathbf{A}_{F} \tilde{\mathbf{B}}_{1}^{T} \mid \mathbf{Y}, \boldsymbol{\Theta}_{-\mathbf{B}_{1}}\right) \\
& =\left(\mathbf{H}_{1}^{T} \mathbf{H}_{1}\right)^{-1} \mathbf{H}_{1}^{T} \mathbf{A}_{F} \mathbf{A}_{F}^{T} \mathbf{Y}=\left(\mathbf{H}_{1}^{T} \mathbf{H}_{1}\right)^{-1} \mathbf{H}_{1}^{T} \mathbf{Y}
\end{aligned}
$$

Note $\mathbf{B}_{1}=\left(\mathbf{H}_{1}^{T} \mathbf{H}_{1}\right)^{-1} \mathbf{H}_{1}^{T} \mathbf{A}_{F} \tilde{\mathbf{B}}_{1}^{T}$, one has

$$
\mathbf{B}_{1}-\hat{\mathbf{B}}_{1}=\left(\mathbf{H}_{1}^{T} \mathbf{H}_{1}\right)^{-1} \mathbf{H}_{1}^{T} \mathbf{A}_{F}\left(\tilde{\mathbf{B}}_{1,0}\right)^{T}=\left(\mathbf{H}_{1}^{T} \mathbf{H}_{1}\right)^{-1} \mathbf{H}_{1}^{T}\left(\mathbf{A}_{s} \tilde{\mathbf{B}}_{1,0, s}^{T}+\mathbf{A}_{c} \tilde{\mathbf{B}}_{1,0, c}^{T}\right)
$$

where $\tilde{\mathbf{B}}_{1,0, s}$ is a $n_{2} \times d$ matrix with the $l$ th column independently sampled from $\mathcal{N}\left(\mathbf{0}, \tilde{\boldsymbol{\Sigma}}_{l}\right)$ for $l=1, \ldots, d$. For the distribution of $\mathbf{A}_{c} \tilde{\mathbf{B}}_{1,0, c}^{T}$, using part 1 of Lemma $\mathrm{S} 1$, we have

$$
\begin{aligned}
p\left(\mathbf{A}_{c} \tilde{\mathbf{B}}_{1,0, c}^{T} \mid \mathbf{Y}, \boldsymbol{\Theta}_{-\mathbf{B}_{1}}\right) & \propto \exp \left\{-\frac{1}{2 \sigma_{0}^{2}} \operatorname{tr}\left(\mathbf{A}_{c}^{T} \tilde{\mathbf{B}}_{1,0, c} \tilde{\mathbf{B}}_{1,0, c}^{T} \mathbf{A}_{c}\right)\right\} \\
& \propto \exp \left\{-\frac{1}{2 \sigma_{0}^{2}} \operatorname{tr}\left(\left(\mathbf{I}_{n_{1}}-\mathbf{A}_{s} \mathbf{A}_{s}^{T}\right) \tilde{\mathbf{B}}_{1,0, c} \tilde{\mathbf{B}}_{1,0, c}^{T}\right)\right\} .
\end{aligned}
$$

Thus we can sample $\mathbf{A}_{c} \tilde{\mathbf{B}}_{1,0, c}^{T}$ by $\sigma_{0}\left(\mathbf{I}_{n_{1}}-\mathbf{A}_{s} \mathbf{A}_{s}^{T}\right) \mathbf{Z}_{0,1}$, where $\mathbf{Z}_{0,1}$ is an $n_{1} \times n_{2}$ matrix with each entry independently sampled from standard normal distribution. The results soon follow.

2. (Column regression coefficients).

We first compute the posterior mean of $\left(\mathbf{B}_{2} \mid \mathbf{Y}, \boldsymbol{\Theta}_{-\mathbf{B}_{2}}\right)$ below

$$
\begin{aligned}
\hat{\mathbf{B}}_{2} & =\mathbb{E}\left(\mathbf{B}_{2} \mid \mathbf{Y}, \boldsymbol{\Theta}_{-\mathbf{B}_{2}}\right)=\mathbb{E}\left(\tilde{\mathbf{B}}_{2} \mathbf{A}_{F}^{T} \mid \mathbf{Y}, \boldsymbol{\Theta}_{-\mathbf{B}_{\mathbf{2}}}\right) \\
& =\sum_{l=1}^{d}\left(\mathbf{H}_{2}^{T} \tilde{\boldsymbol{\Sigma}}_{l}^{-1} \mathbf{H}_{2}\right)^{-1} \mathbf{H}_{2}^{T} \tilde{\boldsymbol{\Sigma}}_{l}^{-1} \mathbf{Y}^{T} \mathbf{a}_{l} \mathbf{a}_{l}^{T}+\left(\mathbf{H}_{2}^{T} \mathbf{H}_{2}\right)^{-1} \mathbf{H}_{2}^{T} \mathbf{Y}^{T}\left(\mathbf{I}_{n_{1}}-\mathbf{A}_{s} \mathbf{A}_{s}^{T}\right)
\end{aligned}
$$


We denote the centered $\tilde{\mathbf{B}}_{2}$ by $\tilde{\mathbf{B}}_{2,0}=\left[\tilde{\mathbf{B}}_{2,0, s}, \tilde{\mathbf{B}}_{2,0, c}\right]$. We have

$$
\mathbf{B}_{2}-\hat{\mathbf{B}}_{2}=\tilde{\mathbf{B}}_{2,0} \mathbf{A}_{F}^{T}=\tilde{\mathbf{B}}_{2,0, s} \mathbf{A}_{s}^{T}+\tilde{\mathbf{B}}_{2,0, c} \mathbf{A}_{c}^{T}
$$

where $\tilde{\mathbf{B}}_{2,0, s}$ is a $q_{2} \times d$ matrix with the $l$ th column independently sampled from $\mathcal{N}\left(\mathbf{0},\left(\mathbf{H}_{2}^{T} \tilde{\boldsymbol{\Sigma}}_{l}^{-1} \mathbf{H}_{2}\right)^{-1}\right)$ for $l=1, \ldots, d$. For the distribution of $\tilde{\mathbf{B}}_{2,0, c} \mathbf{A}_{c}^{T}$, we have

$$
\begin{aligned}
p\left(\tilde{\mathbf{B}}_{2,0, c} \mathbf{A}_{c}^{T} \mid \mathbf{Y}, \boldsymbol{\Theta}_{-\mathbf{B}_{2}}\right) & \propto \exp \left\{-\frac{1}{2 \sigma_{0}^{2}} \operatorname{tr}\left(\mathbf{A}_{c} \mathbf{A}_{c}^{T} \tilde{\mathbf{B}}_{2,0, c} \mathbf{H}_{2}^{T} \mathbf{H}_{2} \tilde{\mathbf{B}}_{2,0, c}^{T}\right)\right\} \\
& \propto \exp \left\{-\frac{1}{2 \sigma_{0}^{2}} \operatorname{tr}\left(\left(\mathbf{I}_{n_{1}}-\mathbf{A}_{s} \mathbf{A}_{s}^{T}\right) \tilde{\mathbf{B}}_{2,0, c} \mathbf{H}_{2}^{T} \mathbf{H}_{2} \tilde{\mathbf{B}}_{2,0, c}^{T}\right)\right\} .
\end{aligned}
$$

Thus we can sample $\tilde{\mathbf{B}}_{2,0, c} \mathbf{A}_{c}^{T}$ by $\sigma_{0}\left(\mathbf{I}_{n_{1}}-\mathbf{A}_{s} \mathbf{A}_{s}^{T}\right) \mathbf{Z}_{0,2}^{T} \mathbf{L}_{H_{2}}^{T}$, where $\mathbf{L}_{H_{2}}$ is a $q_{2} \times q_{2}$ matrix such that $\mathbf{L}_{H_{2}} \mathbf{L}_{H_{2}}^{T}=\left(\mathbf{H}_{2}^{T} \mathbf{H}_{2}\right)^{-1}$ and $\mathbf{Z}_{0,2}$ is a $q_{2} \times n_{1}$ matrix with each entry independently sampled from standard normal distribution.

Lemma S2. Assume $\mathbf{M}=\mathbf{H}_{1} \mathbf{B}_{1}+\left(\mathbf{H}_{2} \mathbf{B}_{2}\right)^{T}$ and let the objective prior $\pi\left(\mathbf{B}_{1}, \mathbf{B}_{2}\right) \propto 1$ for the regression parameters $\mathbf{B}_{1}$ and $\mathbf{B}_{2}$. Denote $\tilde{\mathbf{B}}_{1}=\left[\tilde{\mathbf{b}}_{1,1}, \ldots, \tilde{\mathbf{b}}_{1, n_{1}}\right]=\mathbf{B}_{1}^{T} \mathbf{H}_{1}^{T} \mathbf{A}_{F}$ and $\tilde{\mathbf{B}}_{2}=\left[\tilde{\mathbf{b}}_{2,1}, \ldots, \tilde{\mathbf{b}}_{2, n_{1}}\right]=\mathbf{B}_{2} \mathbf{A}_{F}$.

1. After marginalizing out $\mathbf{Z}$ and $\mathbf{B}_{1}$, assume the marginal posterior distribution of $\tilde{\mathbf{B}}_{1}$ follows

$$
p\left(\tilde{\mathbf{B}}_{1} \mid \mathbf{Y}, \boldsymbol{\Theta}_{-\mathbf{B}_{1},-\mathbf{B}_{2}}\right)=\prod_{l=1}^{n_{1}} \mathcal{P} \mathcal{N}\left(\tilde{\boldsymbol{b}}_{1, l} ; \tilde{\mathbf{y}}_{l}, \mathbf{Q}_{1, l}\right) .
$$

where $\mathbf{Q}_{1, l}=\mathbf{P}_{l}^{T}\left(\tilde{\boldsymbol{\Sigma}}_{l}\right)^{-1} \mathbf{P}_{l}, \mathbf{P}_{l}=\mathbf{I}_{n_{2}}-\mathbf{H}_{2}\left(\mathbf{H}_{2}^{T} \tilde{\boldsymbol{\Sigma}}_{l}^{-1} \mathbf{H}_{2}\right)^{-1} \mathbf{H}_{2}^{T} \tilde{\boldsymbol{\Sigma}}_{l}^{-1}$ for $l=1, \ldots, d$ and $\mathbf{Q}_{1, l}=\sigma_{0}^{2} \mathbf{P}_{0}$ with $\mathbf{P}_{0}=\left(\mathbf{I}_{n_{2}}-\mathbf{H}_{2}\left(\mathbf{H}_{2}^{T} \mathbf{H}_{2}\right)^{-1} \mathbf{H}_{2}^{T}\right)$ for $l=d+1, \ldots, n_{1}$. The sample $\left(\mathbf{B}_{1} \mid \mathbf{Y}, \boldsymbol{\Theta}_{-\mathbf{B}_{1},-\mathbf{B}_{2}}\right)$ can be obtained by $\left(\mathbf{H}_{1}^{T} \mathbf{H}_{1}\right)^{-1} \mathbf{H}_{1}^{T} \mathbf{A}_{F} \tilde{\mathbf{B}}_{1}^{T}$, where $\tilde{\mathbf{B}}_{1}$ sampled from the $p\left(\tilde{\mathbf{B}}_{1} \mid \mathbf{Y}, \boldsymbol{\Theta}_{-\mathbf{B}_{1},-\mathbf{B}_{2}}\right)$ in equation (S5).

2. After marginalizing out $\mathbf{Z}$ and conditional on $\mathbf{B}_{1}$, the marginal posterior distribution of $\tilde{\mathbf{B}}_{2}$ follows (S4) by replacing $\tilde{\mathbf{y}}_{l}$ by $\tilde{\mathbf{y}}_{l, B_{1}}=\left(\mathbf{Y}-\mathbf{H}_{1} \mathbf{B}_{1}\right)^{T} \mathbf{a}_{l}$ for $l=1, \ldots, d$.

Proof of Lemma S2. Denote $\mathbf{Y}_{0}=\mathbf{Y}-\mathbf{H}_{1} \mathbf{B}_{1}-\mathbf{B}_{2}^{T} \mathbf{H}_{2}^{T}$. Define $\mathbf{G}=\left[\mathbf{g}_{1}, \mathbf{g}_{2}, \ldots, \mathbf{g}_{n_{1}}\right]=$ $\left(\mathbf{Y}-\mathbf{H}_{1} \mathbf{B}_{1}\right)^{T} \mathbf{A}_{F}$. That is, $\mathbf{g}_{l}=\left(\mathbf{Y}-\mathbf{H}_{1} \mathbf{B}_{1}\right)^{T} \mathbf{a}_{l}$.

First we have the joint posterior distribution $\left(\mathbf{B}_{1}, \mathbf{B}_{2} \mid \mathbf{Y}, \boldsymbol{\Theta}_{-\mathbf{B}_{1},-\mathbf{B}_{2}}\right)$

$$
\begin{aligned}
& p\left(\mathbf{B}_{1}, \mathbf{B}_{2} \mid \mathbf{Y}, \boldsymbol{\Theta}_{-\mathbf{B}_{1},-\mathbf{B}_{2}}\right) \\
\propto & \exp \left\{-\frac{1}{2} \sum_{l=1}^{d} \mathbf{a}_{l}^{T} \mathbf{Y}_{0}^{T} \tilde{\boldsymbol{\Sigma}}_{l}^{-1} \mathbf{Y}_{0} \mathbf{a}_{l}-\frac{1}{2 \sigma_{0}^{2}} \sum_{l=d+1}^{n_{1}} \mathbf{a}_{l}^{T} \mathbf{Y}_{0}^{T} \mathbf{Y}_{0} \mathbf{a}_{l}\right\}
\end{aligned}
$$


$\propto \exp \left\{-\frac{1}{2} \sum_{l=1}^{d}\left(\mathbf{g}_{l}-\mathbf{H}_{2} \tilde{\mathbf{b}}_{2, l}\right)^{T} \tilde{\boldsymbol{\Sigma}}_{l}^{-1}\left(\mathbf{g}_{l}-\mathbf{H}_{2} \tilde{\mathbf{b}}_{2, l}\right)-\frac{1}{2 \sigma_{0}^{2}} \sum_{l=d+1}^{n_{1}}\left(\mathbf{g}_{l}-\mathbf{H}_{2} \tilde{\mathbf{b}}_{2, l}\right)^{T}\left(\mathbf{g}_{l}-\mathbf{H}_{2} \tilde{\mathbf{b}}_{2, l}\right)\right\}$,

where $\tilde{\mathbf{b}}_{2, l}$ is a transformation of $\mathbf{B}_{2}$ defined in part 2 in Lemma S1.

After integrating out $\tilde{\mathbf{b}}_{2, l}$ from $p\left(\mathbf{B}_{1}, \mathbf{B}_{2} \mid \mathbf{Y}, \boldsymbol{\Theta}_{-\mathbf{B}_{1},-\mathbf{B}_{2}}\right)$ for $l=1,2 \ldots, n_{1}$, one has

$$
\begin{aligned}
& p\left(\mathbf{B}_{1} \mid \mathbf{Y}, \boldsymbol{\Theta}_{-\mathbf{B}_{1},-\mathbf{B}_{2}}\right) \\
\propto & \exp \left\{-\frac{\sum_{l=1}^{d}\left(\mathbf{g}_{l}-\mathbf{H}_{2} \hat{\mathbf{b}}_{2, l}\right)^{T} \tilde{\boldsymbol{\Sigma}}_{l}^{-1}\left(\mathbf{g}_{l}-\mathbf{H}_{2} \hat{\mathbf{b}}_{2, l}\right)}{2}-\frac{\sum_{l=d+1}^{n_{1}}\left(\mathbf{g}_{l}-\mathbf{H}_{2} \hat{\mathbf{b}}_{2, l}\right)^{T}\left(\mathbf{g}_{l}-\mathbf{H}_{2} \hat{\mathbf{b}}_{2, l}\right)}{2 \sigma_{0}^{2}}\right\} \\
\propto & \exp \left\{-\frac{\sum_{l=1}^{d} \mathbf{g}_{l}^{T} \mathbf{P}_{l}^{T}\left(\tilde{\boldsymbol{\Sigma}}_{l}\right)^{-1} \mathbf{P}_{l} \mathbf{g}_{l}}{2}-\frac{\sum_{l=d+1}^{n_{1}} \mathbf{g}_{l}^{T} \mathbf{P}_{0} \mathbf{g}_{l}}{2 \sigma_{0}^{2}}\right\} \\
\propto & \exp \left\{-\frac{\sum_{l=1}^{n_{1}} \mathbf{g}_{l}^{T} \mathbf{Q}_{1, l} \mathbf{g}_{l}}{2}\right\}
\end{aligned}
$$

Where

$$
\hat{\mathbf{b}}_{2, l}= \begin{cases}\left(\mathbf{H}_{2}^{T} \tilde{\boldsymbol{\Sigma}}_{l}^{-1} \mathbf{H}_{2}\right)^{-1} \mathbf{H}_{c}^{T} \tilde{\boldsymbol{\Sigma}}_{l}^{-1} \mathbf{g}_{l} & l=1,2, \ldots, d \\ \left(\mathbf{H}_{2}^{T} \mathbf{H}_{2}\right)^{-1} \mathbf{H}_{2}^{T} \mathbf{g}_{l} & l=d+1, \ldots, n_{1}\end{cases}
$$

Denote $\mathbf{B}_{1}^{\text {aug }}=\left[\mathbf{B}_{1}^{T}, \tilde{\mathbf{B}}_{1,\left(q_{1}+1\right): n_{1}}\right]^{T}$, where $\tilde{\mathbf{B}}_{1,\left(q_{1}+1\right): n_{1}}$ is the last $n_{1}-q_{1}$ columns of $\tilde{\mathbf{B}}_{1}$. Denote the marginal posterior distribution $p_{\text {trans }}\left(\mathbf{B}_{1}^{T} \mid \mathbf{Y}, \boldsymbol{\Theta}_{-\mathbf{B}_{1},-\mathbf{B}_{2}}\right)$ and $p_{\text {trans }}\left(\mathbf{B}_{1}^{\text {aug }} \mid\right.$ $\left.\mathbf{Y}, \boldsymbol{\Theta}_{-\mathbf{B}_{1},-\mathbf{B}_{2}}\right)$ derived by the transformation of $p\left(\tilde{\mathbf{B}}_{1} \mid \mathbf{Y}, \boldsymbol{\Theta}_{-\mathbf{B}_{1},-\mathbf{B}_{2}}\right)$. One has

$$
\begin{aligned}
p_{\text {trans }}\left(\mathbf{B}_{1}^{T} \mid \mathbf{Y}, \Theta_{-\mathbf{B}_{1},-\mathbf{B}_{2}}\right) & \propto p\left(\mathbf{B}_{1}^{\text {aug }} \mid \mathbf{Y}, \Theta_{-\mathbf{B}_{1},-\mathbf{B}_{2}}\right) \\
& =p\left(\tilde{\mathbf{B}}_{1} \mid \mathbf{Y}, \Theta_{-\mathbf{B}_{1},-\mathbf{B}_{2}}\right)\left|\frac{d \tilde{\mathbf{B}}_{1}}{d \mathbf{B}_{1}^{a u g}}\right| \\
& \propto \exp \left\{-\frac{\sum_{l=1}^{n_{1}} \mathbf{g}_{l}^{T} \mathbf{Q}_{1, l} \mathbf{g}_{l}}{2}\right\}
\end{aligned}
$$

Because $\mathbf{Q}_{1, l}$ is idempotent, i.e. $\mathbf{Q}_{1, l} \mathbf{Q}_{1, l}=\mathbf{Q}_{1, l}$, the Moore-Penrose inverse of $\mathbf{Q}_{1, l}$ is $\mathbf{Q}_{1, l}$ itself. Therefore for $l=1, \ldots, d, \tilde{\mathbf{b}}_{1, l} \mid \mathbf{Y}, \boldsymbol{\Theta}_{-\mathbf{B}_{1},-\mathbf{B}_{2}} \sim \mathcal{M}\left(\tilde{\mathbf{y}}_{l}, \mathbf{Q}_{1, l}\right)$, from which the part 1 follows. Part 2 follows Lemma S1.

We are ready to prove Theorem 2 .

Proof of Theorem 2. By Lemma S2, the posterior mean of $\tilde{\mathbf{B}}_{1} \mid \mathbf{Y}, \boldsymbol{\Theta}_{-B_{1},-B_{2}}$ is $\mathbf{Y}^{T} \mathbf{A}_{F}$, where $\mathbf{A}_{F}:=\left[\mathbf{A}_{s}, \mathbf{A}_{c}\right]$. We denote the centered $\tilde{\mathbf{B}}_{1}$ by $\tilde{\mathbf{B}}_{1,0}=\left[\tilde{\mathbf{B}}_{1, Q}, \tilde{\mathbf{B}}_{1,0, c}\right]=\tilde{\mathbf{B}}_{1}-$ $\mathbf{Y}^{T} \mathbf{A}_{F}$, where $\tilde{\mathbf{B}}_{1, Q}$ is the first $d$ columns of $\tilde{\mathbf{B}}_{1,0}$ and $\tilde{\mathbf{B}}_{1,0, c}$ is the next $\left(n_{1}-d\right)$ columns of $\tilde{\mathbf{B}}_{1,0}$. Then the posterior mean of $\mathbf{B}_{1} \mid \mathbf{Y}, \boldsymbol{\Theta}_{-B_{1},-B_{2}}$ can be calculated below 

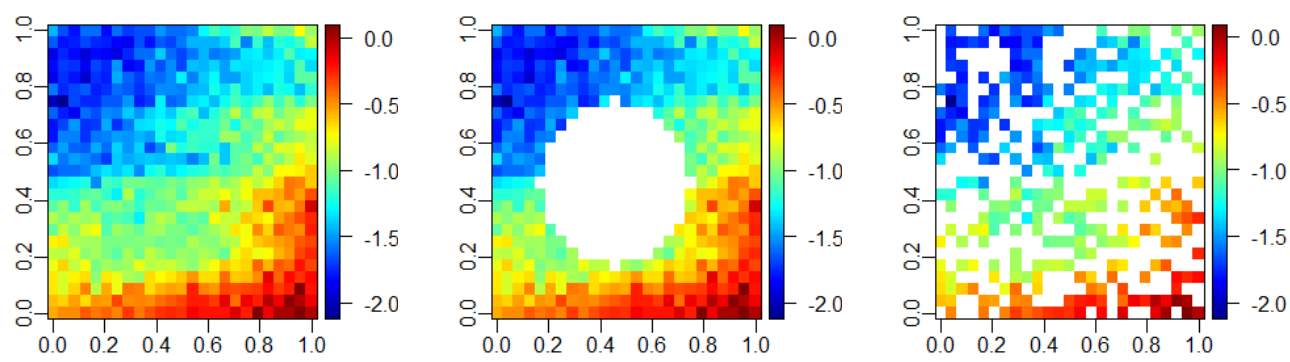

Figure S1: The simulated data with full observations, disk missing pattern and missingat-random pattern with $50 \%$ of the missing values are graphed in the left, middle and right panels, respectively.

$$
\begin{aligned}
\hat{\mathbf{B}}_{1} & =\mathbb{E}\left(\mathbf{B}_{1} \mid \mathbf{Y}, \boldsymbol{\Theta}_{-B_{1},-B_{2}}\right)=\mathbb{E}\left(\left(\mathbf{H}_{1}^{T} \mathbf{H}_{1}\right)^{-1} \mathbf{H}_{1}^{T} \mathbf{A}_{F} \tilde{\mathbf{B}}_{1}^{T} \mid \mathbf{Y}, \boldsymbol{\Theta}_{-B_{1},-B_{2}}\right) \\
& =\left(\mathbf{H}_{1}^{T} \mathbf{H}_{1}\right)^{-1} \mathbf{H}_{1}^{T} \mathbf{A}_{F} \mathbf{A}_{F}^{T} \mathbf{Y}=\left(\mathbf{H}_{1}^{T} \mathbf{H}_{1}\right)^{-1} \mathbf{H}_{1}^{T} \mathbf{Y}
\end{aligned}
$$

Note $\mathbf{B}_{1}=\left(\mathbf{H}_{1}^{T} \mathbf{H}_{1}\right)^{-1} \mathbf{H}_{1}^{T} \mathbf{A}_{F} \tilde{\mathbf{B}}_{1}^{T}$, one has

$$
\mathbf{B}_{1}-\hat{\mathbf{B}}_{1}=\left(\mathbf{H}_{1}^{T} \mathbf{H}_{1}\right)^{-1} \mathbf{H}_{1}^{T} \mathbf{A}_{F}\left(\tilde{\mathbf{B}}_{1,0}\right)^{T}=\left(\mathbf{H}_{1}^{T} \mathbf{H}_{1}\right)^{-1} \mathbf{H}_{1}^{T}\left(\mathbf{A}_{s}\left(\tilde{\mathbf{B}}_{1, Q}\right)^{T}+\mathbf{A}_{c}\left(\tilde{\mathbf{B}}_{1,0, c}\right)^{T}\right)
$$

where by Lemma S2, $\tilde{\mathbf{B}}_{1, Q}$ is an $n_{2} \times d$ matrix with the $l$ th column independently sampled from $\mathcal{N}\left(\mathbf{0}, \mathbf{Q}_{1, l}\right)$ for $l=1, \ldots, d$. For the distribution of $\mathbf{A}_{c} \tilde{\mathbf{B}}_{1,0, c}^{T}$, using part 1 of Lemma S2, we have

$$
\begin{aligned}
& p\left(\mathbf{A}_{c} \tilde{\mathbf{B}}_{1,0, c}^{T} \mid \mathbf{Y}, \boldsymbol{\Theta}_{-B_{1},-B_{2}}\right) \\
\propto & \exp \left\{\frac{1}{2 \sigma_{0}^{2}} \operatorname{tr}\left(\mathbf{A}_{c} \mathbf{A}_{c}^{T} \tilde{\mathbf{B}}_{1,0, c} \mathbf{P}_{0}\left(\tilde{\mathbf{B}}_{1,0, c}\right)^{T}\right)\right\} \\
\propto & \exp \left\{-\frac{1}{2 \sigma_{0}^{2}} \operatorname{tr}\left(\left(\mathbf{I}_{n_{1}}-\mathbf{A}_{s} \mathbf{A}_{s}^{T}\right) \tilde{\mathbf{B}}_{1,0, c} \mathbf{P}_{0}\left(\tilde{\mathbf{B}}_{1,0, c}\right)^{T}\right)\right\} .
\end{aligned}
$$

Thus we can sample marginal posterior distribution of $\mathbf{A}_{c} \tilde{\mathbf{B}}_{1,0, c}^{T}$ by $\sigma_{0}\left(\mathbf{I}_{n_{1}}-\mathbf{A}_{s} \mathbf{A}_{s}^{T}\right) \mathbf{Z}_{0,1} \mathbf{P}_{0}$, where $\mathbf{Z}_{0,1}$ is an $n_{1} \times n_{2}$ matrix with each entry independently sampled from standard normal distribution. The results soon follow. 

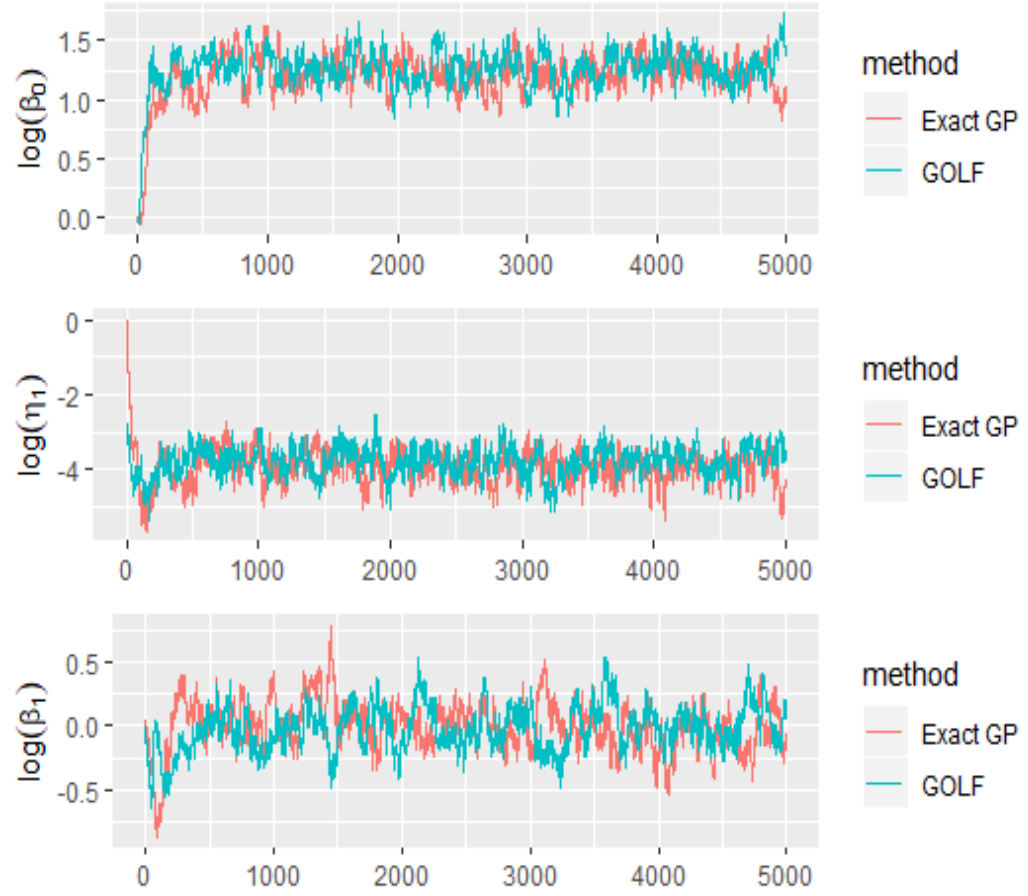

Figure S2: Trace plots of posterior samples of parameters in the exact GP model and GOLF processes for the simulated data in figure S1.

\section{S2 Additional results of simulated studies in Section 5}

We provide additional results for the simulated studies in Example 3 in Figure S1 and Figure S2. We graph the simulated data set with full observations, disk missing pattern and missing-at-random pattern with 50\% of the missing values in Figure S1. Posterior samples of the logarithm of the inverse range parameter of factor loading matrix, the nugget parameter and the inverse range parameter of the factors are graphed from the upper to lower panels in Figure S2, respectively. The posterior samples of parameters in the exact GP model and GOLF processes are similar to each other.

\section{S3 Additional results for real applications in Section 6.1}

In this section, we include additional results for GOLF processes predicting the missing values of the temperature data set discussed in Heaton et al. (2019). We show the details of 5 different configurations of GOLF processes, where the result reported in the main body of the article is the configuration 1. For all the configurations, the proportion of the burn-in samples is $20 \%$. We use the normal distribution centered on the previous values as the proposal distribution of the logarithm of the inverse range parameters and 

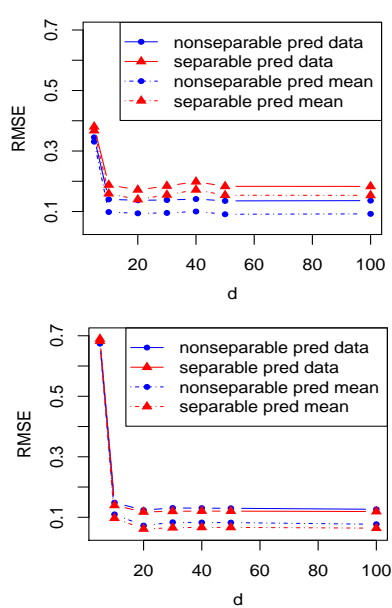
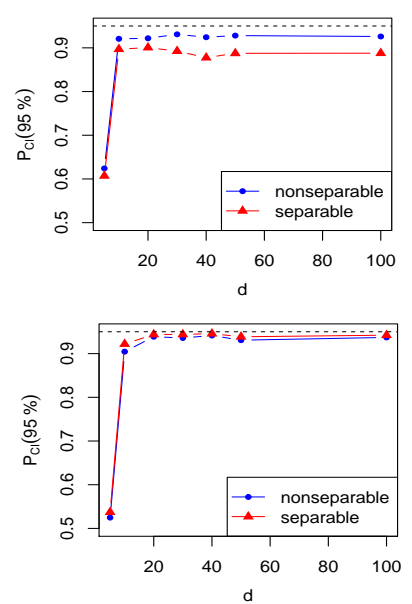
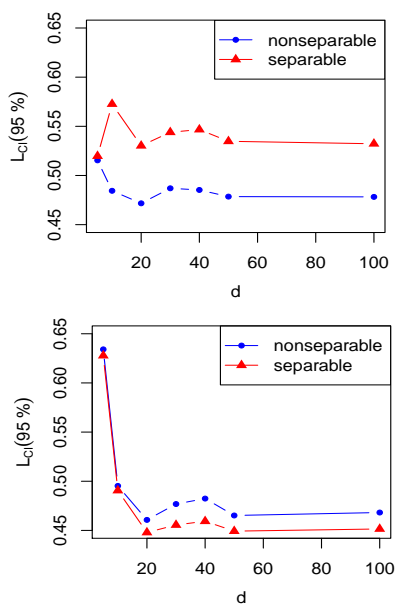

Figure S3: The predictive performance of GOLF process with $d=5,10,20,30,40,50$ and 100 factors for Example 4, when the true number of factor is $d_{\text {real }}=100$ in generating the data. The nonseparable kernel with distinct kernel parameters is assumed to generate the data in the first row of panels, and the separable kernel with the same kernel parameter of each factor process is used for simulation in the second row of panels. The blue curves and red curves denote the performance by the GOLF processes with the different kernel parameters and the same kernel parameter, respectively. In the left panels, the solid curves denote the RMSE for predicting the (noisy) observations, and the dashed curve denotes the RMSE for predicting the mean of the observations. The proportions of observations covered in the $95 \%$ predictive interval and the average length of the predictive interval are graphed in the middle and right panels, respectively.

logarithm of the nugget parameters. For the logarithm of the inverse range parameters of the factor loading matrix, the standard deviation of the proposal distribution is $40 / n_{1}$. For the logarithm of the inverse range parameters and the nugget parameters of the factor processes, the standard deviation of the posterior distribution is set to be $40 / n_{2}$.

The details of 5 configurations are given in Table S1. The predictive RMSE, $P_{C I}(95 \%)$ and $L_{C I}(95 \%)$ of the 5 configurations are given in Table S2. The predictive RMSE is similar for all 5 configurations. Increasing the posterior sample size seems to slightly increase the proportion of the samples contained in the $95 \%$ predictive interval. 


\begin{tabular}{lccccc}
\hline & sample size & system & initial $Y_{v, i}^{*}$ & initial $\log \left(\beta_{0}\right)$ & initial $\log \left(\beta_{l}\right)$ \\
\hline Conf. 1 & 6000 & Mac & mean at each latitude & 3 & 0 \\
Conf. 2 & 6000 & Win & mean at each latitude & 3 & 0 \\
Conf. 3 & 40000 & Mac & mean at each latitude & 3 & 0 \\
Conf. 4 & 40000 & Mac & overall mean + noise & 3 & 0 \\
Conf. 5 & 40000 & Mac & mean at each latitude & Unif[-1,1] & Unif[-1,1] \\
\hline
\end{tabular}

Table S1: Detailed settings of 5 different configurations of GOLF processes for the data set in Heaton et al. (2019). The number of samples and the computing system are shown in the second column and third column, respectively. The choice of the initial values of the missing data is given in the fourth column, using either the mean of the observations at each latitude or overall mean of the observations with a small random Gaussian noise (with standard deviation being 0.1 times of the standard deviation of the observations). The initial values of the logarithm of the inverse range parameters are either chosen to be a fixed value or randomly sampled from the uniform distribution, shown in columns $5-6$.

\begin{tabular}{lccc}
\hline Methods & RMSE & $P_{C I}(95 \%)$ & $L_{C I}(95 \%)$ \\
\hline Configuration 1 & 1.46 & 0.92 & 4.95 \\
Configuration 2 & 1.50 & 0.91 & 4.92 \\
Configuration 3 & 1.44 & 0.94 & 7.70 \\
Configuration 4 & 1.48 & 0.94 & 7.75 \\
Configuration 5 & 1.51 & 0.93 & 5.16 \\
\hline
\end{tabular}

Table S2: Predictive performance of 5 different implementations for the data set in Heaton et al. (2019).
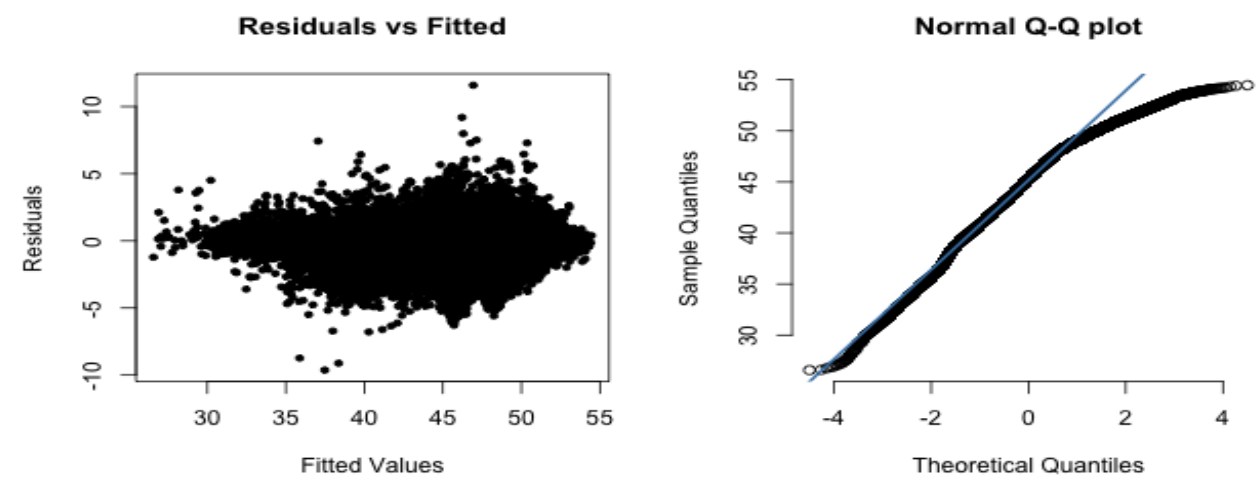

Figure S4: Diagnostic plots of the GOLF processes for the data set in Heaton et al. (2019).

The fitted values from the GOLF processes in configuration 1 against the residuals 
and the normal Q-Q plot are graphed in the left panel and the right panel in Figure S4, respectively. The Q-Q plot indicates the fitted values are slightly left-skewed and slightly under-dispersed.

\section{References}

$\mathrm{Gu}$, M. and Shen, W. (2020). "Generalized probabilistic principal component analysis of correlated data." Journal of Machine Learning Research, 21(13).

Heaton, M. J., Datta, A., Finley, A. O., Furrer, R., Guinness, J., Guhaniyogi, R., Gerber, F., Gramacy, R. B., Hammerling, D., Katzfuss, M., et al. (2019). "A case study competition among methods for analyzing large spatial data." Journal of Agricultural, Biological and Environmental Statistics, 24(3): 398-425. 